\title{
Survey on pain in patients with chronic skin lesions
}

Ciro Falasconi, Vincenzo Amalfi, Patrizia Baroni, Giovanni Vito Corona, Corrado Maria Durante, Paola Fanin, Caterina Favaro, Massimo Fornaciari, Alessandro Farris, Manuela Galleazzi, Francesco Giacinto, Giorgio Guarnera, Vincenzo Lauletta, Mario Marazzi, Marco Masina, Vincenzo Mattaliano, Giovanni Battista Mosti, Giuseppe Nebbioso, Francesco Stanganello, Francesco Petrella

Italian Association of Skin Ulcers, Turin, Italy

\section{INTRODUCTION}

In the three months period between March and June 2016 we launched a survey on the AUIC website, open to the contribution of all members, to evaluate the most widespread habits and the most common practices (for therapy and overall healthcare) concerning pain management for patients with chronic skin lesions (CSL).

We decided to evaluate this aspect of our clinical practice because we believe that the problems connected to "pain" in patients affected by CSL often becomes an issue even larger than the ulcer itself for both patients and their families. Furthermore, the theme "pain" is strongly felt at all levels; the body of knowledge in its regard is increasingly large and widespread and the means at our disposal to address this particular aspect of the care of CSL are continually increasing. For these reasons it seemed fair to focus the attention of all members on this clinical and healthcare problem, in order to collect useful information to improve the overall quality of care for our patients.

Correspondence: Italian Association of Skin Ulcers, Via San Francesco da Paola 37, 10123 Turin, Italy.

Tel: +39.011.2446911 - Fax: +39.011.2446950.

E-mail: info@aiuc.it

Key words: Chronic skin lesions; Pain management; Survey; Italian Association of Skin Ulcers.

Received for publication: 31 December 2016.

Accepted for publication: 7 March 2017.

This work is licensed under a Creative Commons Attribution NonCommercial 4.0 License (CC BY-NC 4.0).

(C) Copyright C. Falasconi et al., 2017

Licensee PAGEPress, Italy

Italian Journal of Wound Care 2017; 1(1):19-26

doi:10.4081/ijwc.2017.3

\section{MATERIALS AND METHODS}

The survey was carried out using the SurveyMonkey platform which presented a questionnaire with 26 multiple choice questions to the members who wanted to participate. The questionnaire, which required about 15 minutes, was anonymous and there was no way to trace the data of the participants.

For some questions, which had very narrow implications related to drug therapies, the participant was given the opportunity to indicate their status as a physician or as belonging to a different health profession, so as to avoid answering, avoiding questions of competence.

\section{RESULTS}

The survey was filled out by 118 members and while nearly all of them (83\%) observe it in the great majority of cases, almost all of them (99\%) consider pain symptoms as very relevant for patients with CSL (Figure 1).

The vast majority of participants use validated pain assessment scales with patients with impaired verbal communication (Figure 2A and B).

Underlying and procedural pain are held in great consideration, especially in arterial, vasculitic and neuropathic ulcers, which are considered the most painful (Figure $3 \mathrm{~A}$ and $\mathrm{B}$ ).

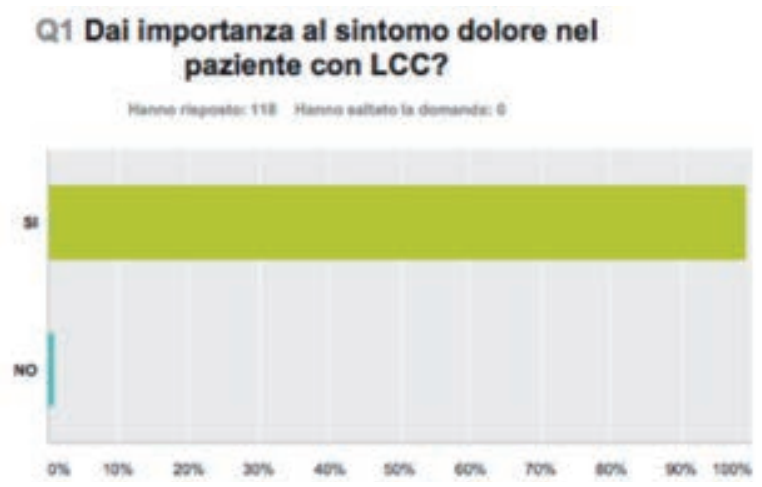

Figure 1. 
Q3 Se SI, come valuti tale sintomo?
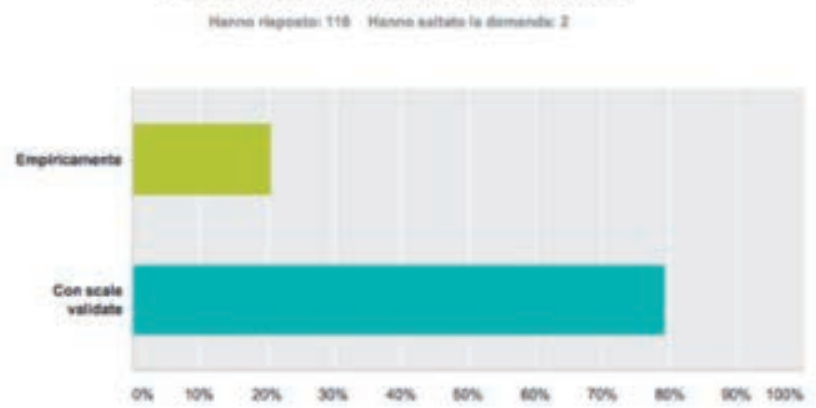

Q4 Valuti il dolore nel paziente con ridotta capacità di comunicazione verbale?

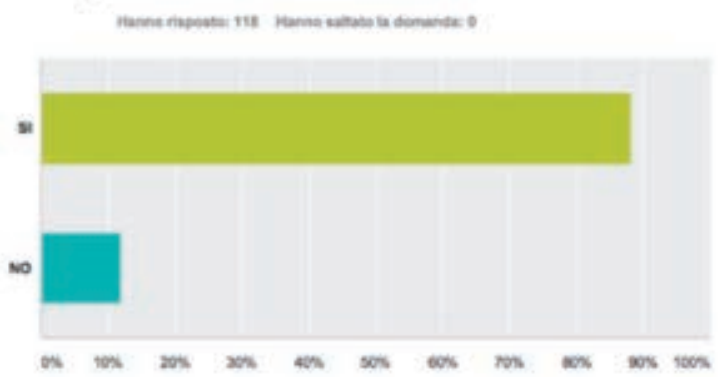

Figure 2.

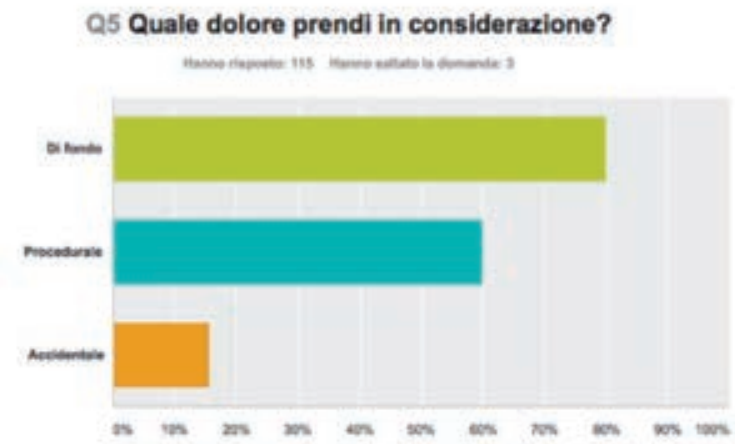

Figure 3.

Infection and, secondly, the presence of necrotic material on the bed, are believed to be conditions that can cause a significant increase in pain in these patients (Figure 4).

Pain medications are routinely used by approximately $95 \%$ of operators.

The majority use these medicines regularly to prevent the onset of pain or to reduce its degree.

Of participating doctors $75 \%$ consider the major opioids, acetaminophen and corticosteroids as the first treatment option. NSAIDs are considered as the second option by about $50 \%$ of operators, while minor opioids are used as a the third option by more than $35 \%$ (Figure 5).

More than $95 \%$ of participants consider the process of dressing change as a major cause of pain to which they dedicate much attention (Figure 6A and B).

About $70 \%$ of operators consider the dressing itself as a

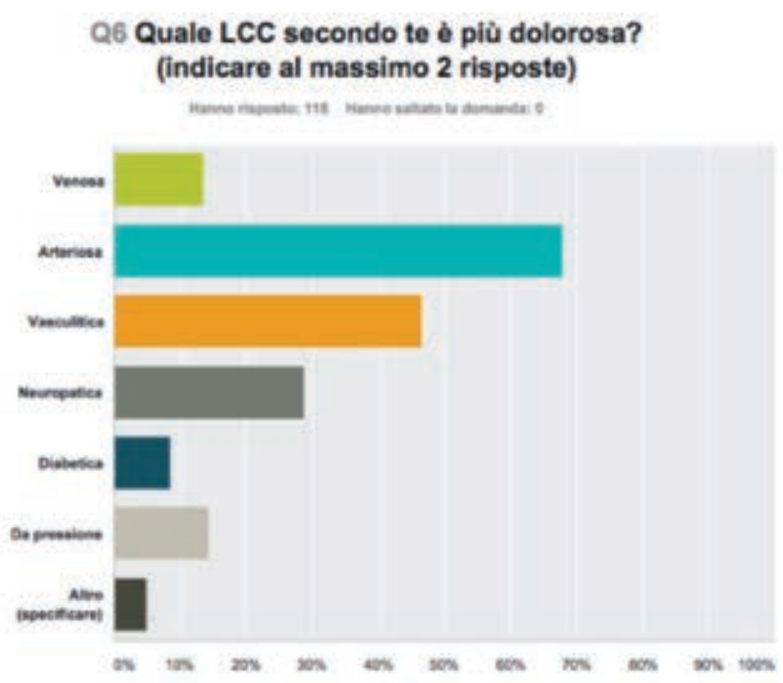

Q6 Quale LCC secondo te è più dolorosa? (indicare al massimo 2 risposte)

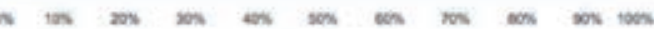


potential source of pain and the status of the surrounding skin is considered by almost everyone as a decisive factor for the worsening of the painful condition (Figure 7A and B).

A minority of operators (approximately 25\%) use medications that contain NSAIDS for controlling CSL pain, while the underlying or neuropathic pain and the procedural pain are treated using local anesthetics respectively by $50 \%$ and $75 \%$ of physicians (Figure 8 ).

\begin{tabular}{|c|c|c|c|c|c|c|c|}
\hline & 1 & 2 & 3 & 4 & 5 & Totale & Punteggio \\
\hline Paracelamodo & $\begin{array}{r}25,81 \% \\
8\end{array}$ & $2.23 \%$ & $\begin{array}{r}12.90 \% \\
4\end{array}$ & $\begin{array}{r}25,81 \% \\
8\end{array}$ & $\begin{array}{r}32.26 \% \\
10\end{array}$ & 31 & 265 \\
\hline Fans & $\begin{array}{r}10,71 \% \\
3\end{array}$ & $\begin{array}{r}46,43 \% \\
13\end{array}$ & $21,49 \%$ & $\begin{array}{r}10,71 \% \\
3\end{array}$ & $\begin{array}{r}10,71 \% \\
3\end{array}$ & 28 & 3.36 \\
\hline Cortisonid & $\begin{array}{r}25,00 \% \\
5\end{array}$ & $\begin{array}{r}20,00 \% \\
4\end{array}$ & $\begin{array}{r}35,00 \% \\
7\end{array}$ & $\begin{array}{r}15.00 \% \\
3\end{array}$ & $\begin{array}{r}5.00 \% \\
1\end{array}$ & 20 & 3,45 \\
\hline Oppibia minon & $\begin{array}{r}11,11 \% \\
4\end{array}$ & $\begin{array}{r}16,6 \mathrm{rs} \\
6\end{array}$ & $\begin{array}{r}36,11 \% \\
13\end{array}$ & $\begin{array}{r}25.00 \% \\
9\end{array}$ & $\begin{array}{r}11,11 \% \\
4\end{array}$ & 30 & 292 \\
\hline Oppioid magjion & $\begin{array}{r}30,00 \% \\
9\end{array}$ & $\begin{array}{r}23,33 \% \\
7\end{array}$ & $\begin{array}{r}10,00 \% \\
3\end{array}$ & $\begin{array}{r}16.67 \% \\
5\end{array}$ & $\begin{array}{r}20,00 \% \\
6\end{array}$ & 30 & 3.27 \\
\hline
\end{tabular}

Figure 5.
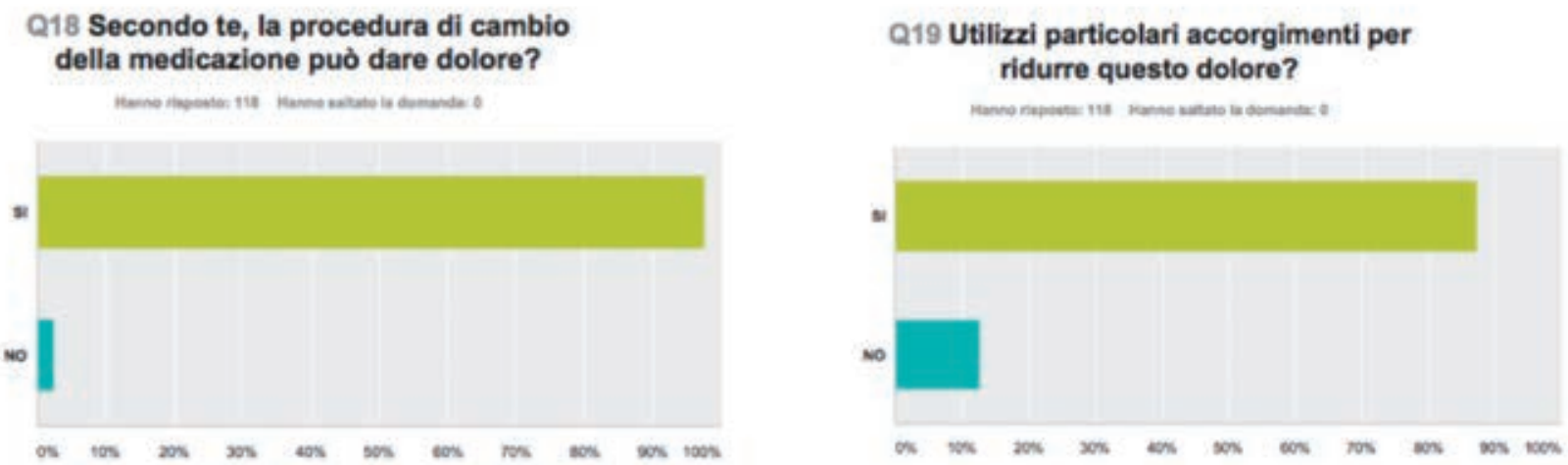

Figure 6.

\section{Q21 La scelta della medicazione condiziona il dolore al cambio della stessa?}

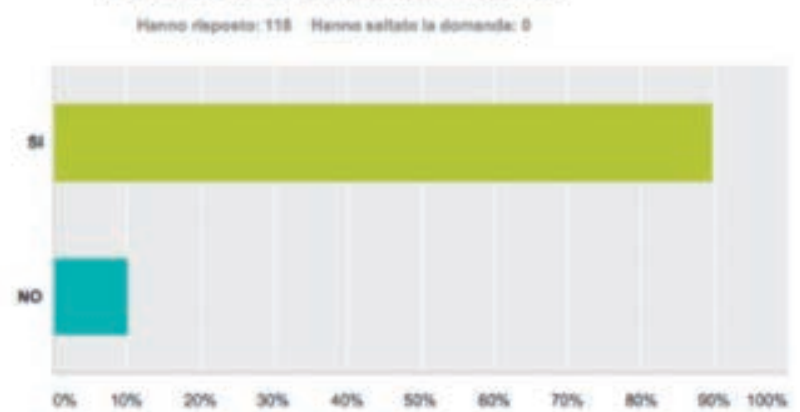

\section{Q23 Lo stato della cute perilesionale condiziona il dolore della LCC?}

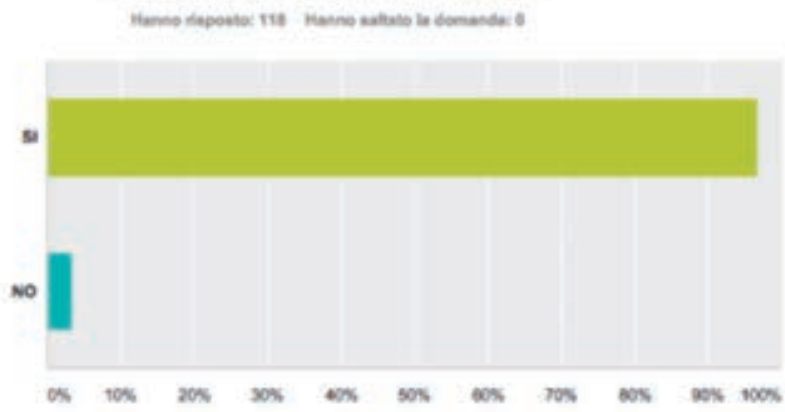

Figure 7. 


\section{Q24 Utilizzi medicazioni che contengono} fans?

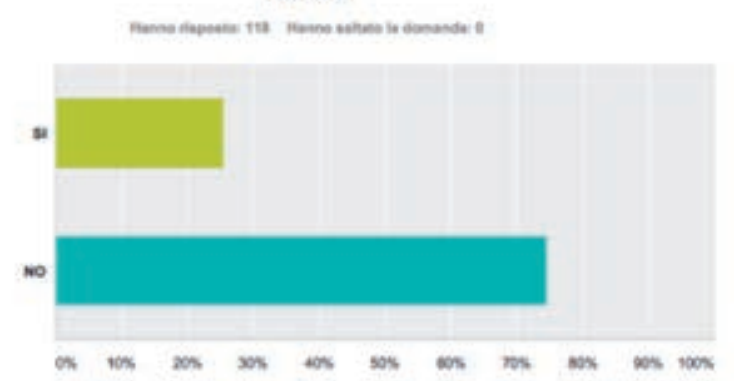

Figure 8.

\section{CONCLUSIONS}

We can say that the responses obtained by our survey are perfectly in line with the current and most widespread clinical guidelines and best practices.

The use of pain medications is very relevant and their use demonstrates the great sensitivity of health professionals involved in wound care towards their patients whose issue is considered on par with much more challenging clinical problems.

Similarly, it is commendable to see how infection is considered as a cause of pain and how the vast majority of participants recognize antibiotic therapy as a tool for controlling pain other than dressings and analgesics.

Evidently, the scarce use of medications containing NSAIDs shows how perhaps we need further studies in this particular area in order to more completely assess if this treatment option can really be another tool for CSL pain control or if the use of such topical drugs represents a way to accelerate a healing process altered by chronic inflammation. 


\section{Indagine conoscitiva sul dolore nel paziente con le lesioni croniche cutanee}

Ciro Falasconi, Vincenzo Amalfi, Patrizia Baroni, Giovanni Vito Corona, Corrado Maria Durante, Paola Fanin, Caterina Favaro, Massimo Fornaciari, Alessandro Farris, Manuela Galleazzi, Francesco Giacinto, Giorgio Guarnera, Vincenzo Lauletta, Mario Marazzi, Marco Masina, Vincenzo Mattaliano, Giovanni Battista Mosti, Giuseppe Nebbioso, Francesco Stanganello, Francesco Petrella

Associazione Italiana Ulcere Cutanee

\section{INTRODUZIONE}

Nel trimestre marzo-giugno 2016 abbiamo avviato sul sito AIUC una indagine conoscitiva, aperta al contributo di tutti i soci, per valutare le abitudini più diffuse ed i più comuni orientamenti (terapeutici ed assistenziali) in tema di gestione del dolore nei pazienti con lesioni croniche cutanee (LCC).

Abbiamo deciso di valutare questo aspetto della nostra pratica clinica perché convinti che il problema "dolore" nel paziente con LCC abbia spesso un peso, sia per il paziente che per il suo cordone familiare, anche maggiore dell'ulcera stessa. Inoltre, il tema "dolore" è molto sentito a tutti i livelli, le conoscenze in materia sono sempre più ampie e diffuse ed i mezzi a nostra disposizione per affrontare questo particolare aspetto della cura delle LCC aumentano continuamente. Per questi motivi ci è sembrato giusto focalizzare l'attenzione di tutti i soci su questo problema clinico ed assistenziale, al fine di raccogliere informazioni utili per migliorare complessivamente la qualità delle cure rivolte ai nostri pazienti.

\section{MATERIALI E METODI}

L'indagine è stata realizzata utilizzando la piattaforma SurveyMonkey e somministrando ai soci che volevano partecipare un questionario con 26 domande a risposta multipla. La compilazione del questionario, che richiedeva un tempo di circa 15 minuti, è stata realizzata in forma anonima e in nessun modo è possibile risalire ai dati dei partecipanti.

Per alcune domande, con delle strettissime implicazioni legate alle terapie farmacologiche, è stata data al partecipante la possibilità di indicare il suo stato di medico o di appartenente a diversa professione sanitaria, in modo da non rispondere, evitando problemi di competenza.

\section{RISULTATI}

Hanno risposto alla survey 118 soci e la quasi tota- lità (oltre il 99\%) considera importante il sintomo dolore nei pazienti con LCC e lo riscontra quasi sempre (83\%) (Figura 1).

La grande maggioranza dei partecipanti utilizza scale validate di valutazione del sintomo dolore anche nei pazienti con ridotta capacità di comunicazione verbale (Figura $2 \mathrm{~A}$ e $\mathrm{B}$ ).

Il dolore di fondo e quello procedurale sono tenuti in grande considerazione, soprattutto nelle ulcere arteriose, vasculitiche e neuropatiche che sono considerate, complessivamente, come le più dolorose (Figura $3 \mathrm{~A}$ e $\mathrm{B}$ ).

L'infezione e, in seconda battuta, la presenza di materiale necrotico sul fondo, sono ritenute le condizioni in grado di determinare un sensibile aumento del dolore in questi pazienti (Figura 4).

I farmaci antidolorifici sono regolarmente utilizzati da circa il 95\% degli operatori.

La maggior parte utilizza tali farmaci regolarmente, onde evitare l'insorgenza del dolore o, comunque, per ridurne l'entità.

Il $75 \%$ dei medici partecipanti considera gli oppioidi maggiori, il paracetamolo e i cortisonici come prima opzione terapeutica. I FANS sono considerati in seconda linea da circa il $50 \%$ degli operatori, mentre gli oppioidi minori sono utilizzati in terza linea da oltre il 35\% (Figura 5).

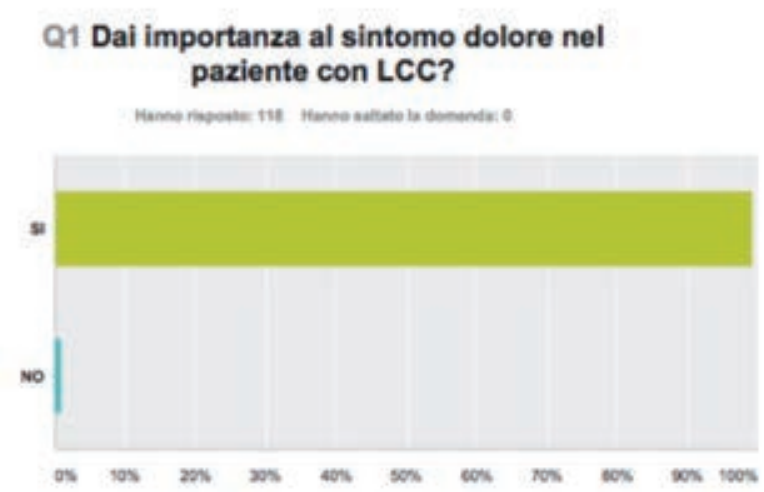

Figura 1. 
Q3 Se SI, come valuti tale sintomo?
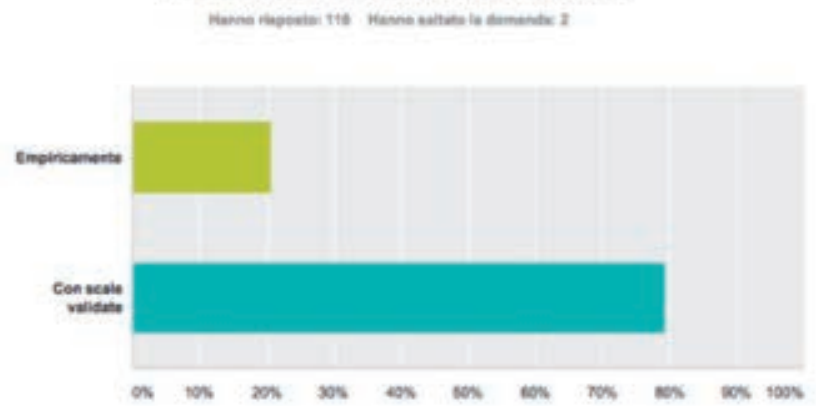

Q4 Valuti il dolore nel paziente con ridotta capacità di comunicazione verbale?

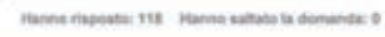

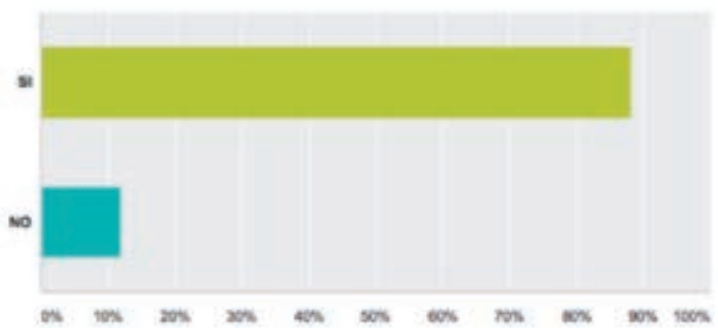

Figura 2.
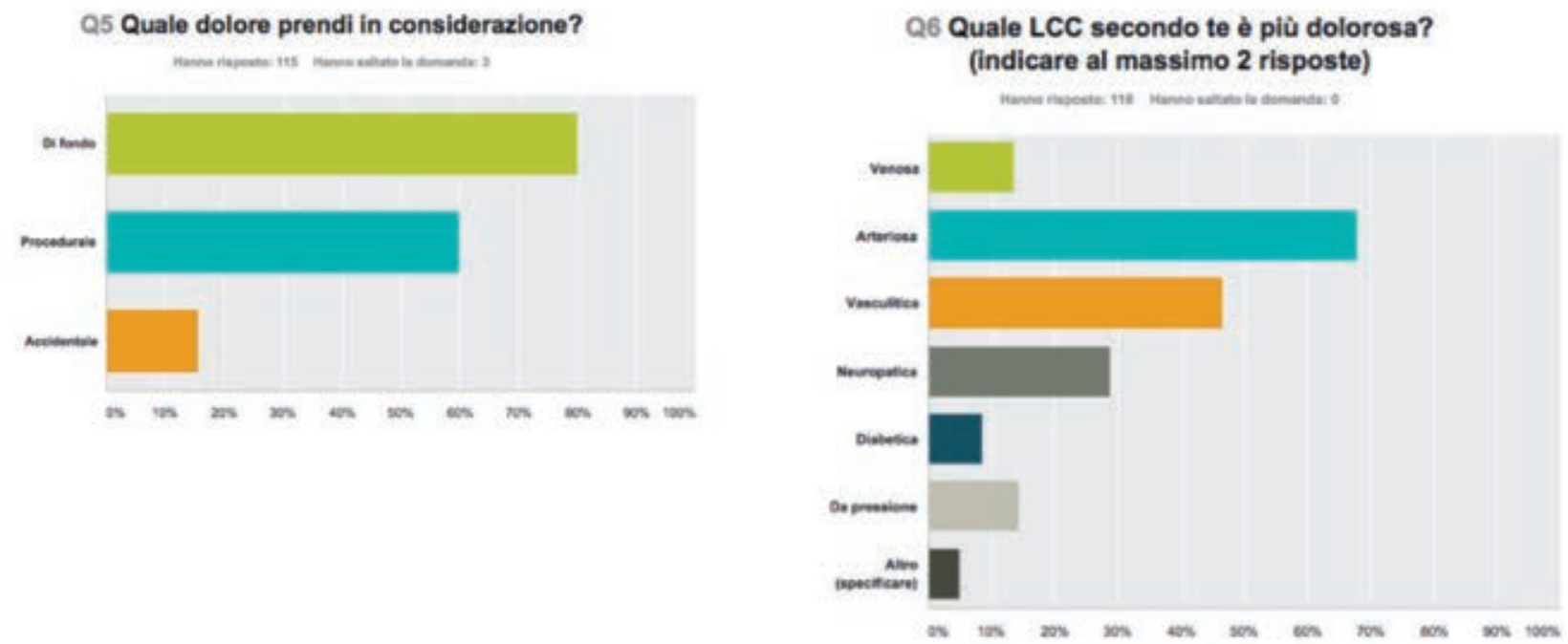

Figura 3.

Sicuramente (da oltre il 95\% dei partecipanti) la procedura di cambio della medicazione è considerata una delle maggiori cause di dolore alla quale viene dedicata molta attenzione (Figura 6A e B).

Per circa il 70\% degli operatori la medicazione in sé rappresenta possibile fonte di dolore e lo stato della cute perilesionale è considerato praticamente da tutti come elemento determinante per il peggioramento della condizione dolorosa (Figura 7A e B).

Una minoranza degli operatori (circa il 25\%) adopera medicazioni che contengono FANS per controllare il dolore delle LCC, mentre il dolore di fondo o neuropatico ed il dolore procedurale sono trattati con anestetici locali rispettivamente da circa il 50 ed il $75 \%$ dei medici (Figura 8).

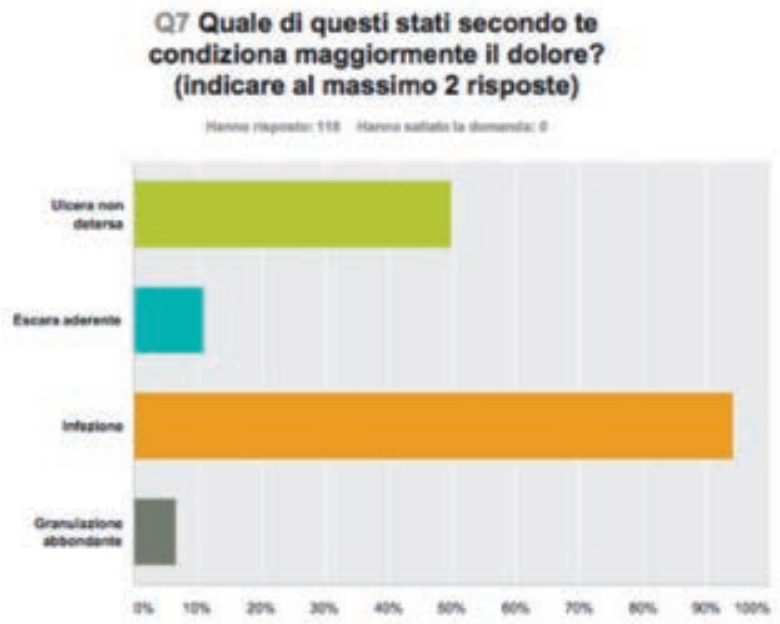

Figura 4. 


\section{CONCLUSIONI}

Possiamo dire che le risposte ottenute dalla nostra indagine conoscitiva sono perfettamente in linea con gli at- tuali e più diffusi orientamenti clinici espressi da documenti di Best Practice e dalle linee guida.

Rilevante è l'utilizzo di farmaci antidolorifici le cui modalità di impiego denotano una grande sensibilità degli

\begin{tabular}{|c|c|c|c|c|c|c|c|}
\hline & 1 & 2 & 3 & 4 & 5 & Totale & Puntegolo \\
\hline Paracelamedo & $\begin{array}{r}25,45 \\
8\end{array}$ & $\begin{array}{r}2.23 \% \\
5\end{array}$ & $\begin{array}{r}12.90 \% 5 \\
4\end{array}$ & $\begin{array}{r}25.81 \% \\
8\end{array}$ & $\begin{array}{r}32.26 \% 5 \\
10\end{array}$ & 31 & 2.65 \\
\hline Fans & $\begin{array}{r}10,71 \% \\
3\end{array}$ & $\begin{array}{r}46,49 \% \\
13\end{array}$ & $21,43 \%$ & $\begin{array}{r}10,71 \% \\
3\end{array}$ & $\begin{array}{r}10,71 \% \\
3\end{array}$ & 28 & 336 \\
\hline Cattinorid: & $\begin{array}{r}25,00 \% \\
5\end{array}$ & $\begin{array}{r}20,00 \% \\
4\end{array}$ & $\begin{array}{r}35,00 \% \\
7\end{array}$ & $\begin{array}{r}15,00 \% \\
3\end{array}$ & $\begin{array}{r}5,00 \% \\
1\end{array}$ & 20 & 3,45 \\
\hline Oppioid minen & $\begin{array}{r}11,11 \% \\
4\end{array}$ & $\begin{array}{r}16,67 \times 5 \\
6\end{array}$ & $\begin{array}{r}36,11 \% \\
13\end{array}$ & $\begin{array}{r}25,00 \% \\
9\end{array}$ & $\begin{array}{r}11,11 \% \\
4\end{array}$ & 35 & 2.92 \\
\hline Cppioid maggion & $\begin{array}{r}30,00 \% \\
9\end{array}$ & $\begin{array}{r}20,33 \% \\
7\end{array}$ & $\begin{array}{r}10,00 \mathrm{~N} \\
3\end{array}$ & $\begin{array}{r}16.67 \% \\
5\end{array}$ & $\begin{array}{r}20,00 \% \\
6\end{array}$ & 30 & 3.27 \\
\hline
\end{tabular}

Figura 5.

\section{Q18 Secondo te, la procedura di cambio della medicazione può dare dolore?}

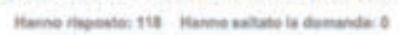

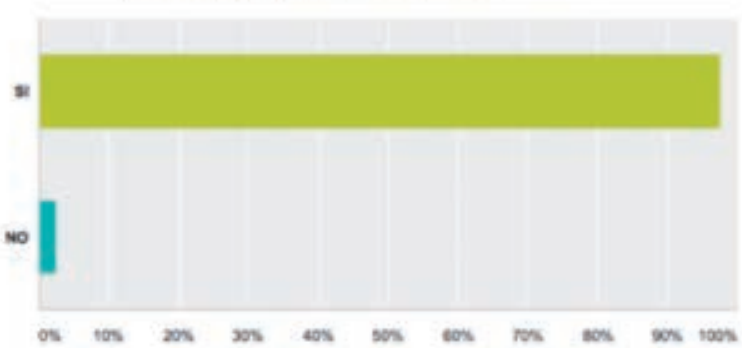

Figura 6.

\section{Q19 Utilizzi particolari accorgimenti per ridurre questo dolore?}

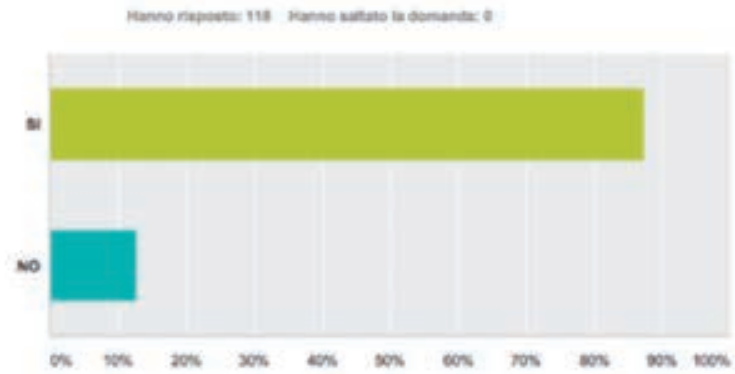

\section{Q21 La scelta della medicazione condiziona il dolore al cambio della stessa?}

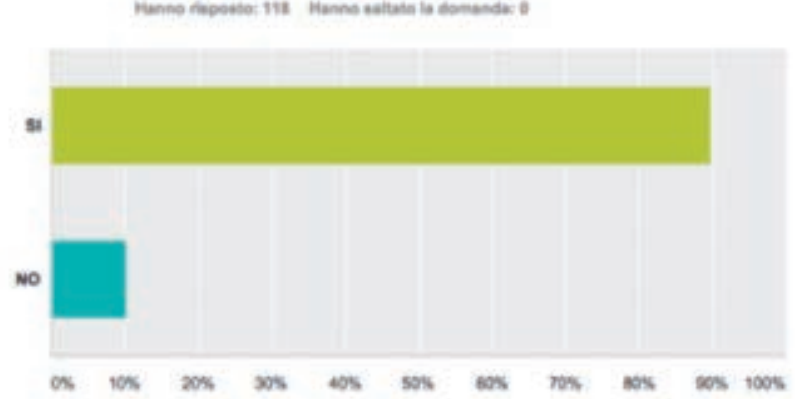

\section{Q23 Lo stato della cute perilesionale} condiziona il dolore della LCC?

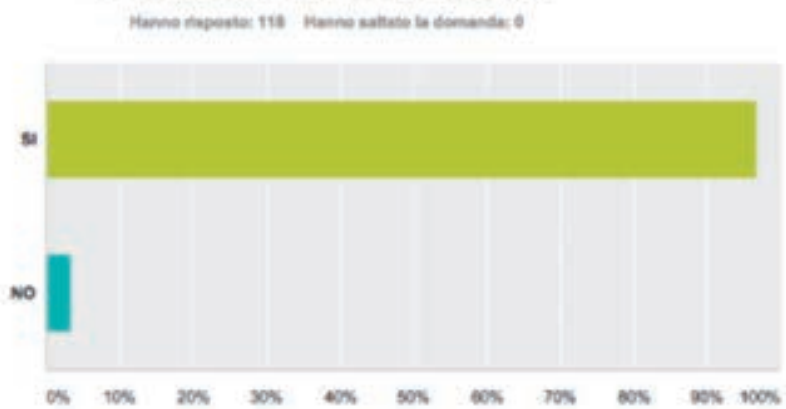

Figura 7. 

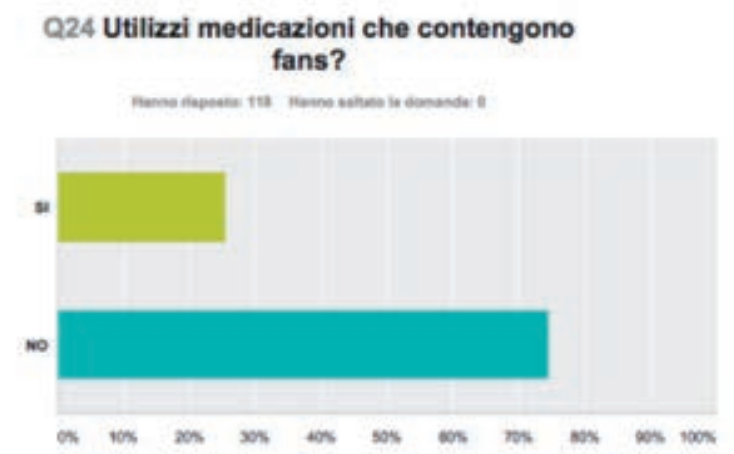

Figura 8. operatori sanitari impegnati nel Wound Care verso i loro pazienti il cui problema viene considerato alla pari di problemi clinici ben più impegnativi.

Allo stesso modo, è apprezzabile la considerazione dell'infezione come causa di dolore e il riconoscimento della terapia antibiotica, da gran parte dei partecipanti, come uno dei mezzi per controllare il dolore diversi dalla medicazione e dagli analgesici.

Evidentemente, lo scarso impiego delle medicazioni contenenti FANS mostra come siano forse necessari ulteriori studi in questo settore specifico per valutare, in modo più completo, se tale opzione terapeutica possa rappresentare veramente una ulteriore modalità di controllo del dolore nelle LCC, oppure se l'impiego di tali farmaci per uso topico rappresenti un mezzo per accelerare i processi di guarigione, alterati dalla infiammazione cronica. 